\title{
REGULARITY AT INFINITY OF HADAMARD MANIFOLDS WITH RESPECT TO SOME ELLIPTIC OPERATORS AND APPLICATIONS TO ASYMPTOTIC DIRICHLET PROBLEMS
}

\author{
JAIME RIPOLL AND MIRIAM TELICHEVESKY
}

Abstract. Let $M$ be Hadamard manifold with sectional curvature $K_{M} \leq$ $-k^{2}, k>0$. Denote by $\partial_{\infty} M$ the asymptotic boundary of $M$. We say that $\bar{M}$ satisfies the strict convexity condition (SC condition) if, given $x \in \partial_{\infty} M$ and a relatively open subset $W \subset \partial_{\infty} M$ containing $x$, there exists a $C^{2}$ open subset $\Omega \subset M$ such that $x \in \operatorname{Int}\left(\partial_{\infty} \Omega\right) \subset W$ and $M \backslash \Omega$ is convex. We prove that the SC condition implies that $M$ is regular at infinity relative to the operator

$$
\mathcal{Q}[u]:=\operatorname{div}\left(\frac{a(|\nabla u|)}{|\nabla u|} \nabla u\right),
$$

subject to some conditions. It follows that under the SC condition, the Dirichlet problem for the minimal hypersurface and the $p$-Laplacian $(p>1)$ equations are solvable for any prescribed continuous asymptotic boundary data. It is also proved that if $M$ is rotationally symmetric or if $\inf _{B_{R+1}} K_{M} \geq$ $-e^{2 k R} / R^{2+2 \epsilon}, R \geq R^{*}$, for some $R^{*}$ and $\epsilon>0$, where $B_{R+1}$ is the geodesic ball with radius $R+1$ centered at a fixed point of $M$, then $M$ satisfies the SC condition.

\section{INTRODUCTION}

Let $M$ be Hadamard manifold (complete, simply connected Riemannian manifold) with sectional curvature $K_{M} \leq-k^{2}, k>0$. Denote by $\bar{M}$ the compactification of $M$ in the cone topology and by $\partial_{\infty} M$ the asymptotic boundary of $M$ (see for instance [EO]). This upper bound hypothesis on the sectional curvature of $M$ will be assumed throughout the paper.

We consider the elliptic diferential operator $\mathcal{Q}$ defined in the Sobolev space $W_{\text {loc }}^{1, p}(M)$, for some $p \geq 1$, given by

$$
\mathcal{Q}[u]:=\operatorname{div}\left(\frac{a(|\nabla u|)}{|\nabla u|} \nabla u\right)=0,
$$

where $a \in C^{1}([0, \infty))$ satisfies

$$
\begin{aligned}
& a(0)=0, a^{\prime}(s)>0 \text { for all } s>0 ; \\
& a(s) \leq C\left(s^{p-1}+1\right) \text { for all } s \in[0,+\infty), \text { for some constant } C>0 \\
& \text { there exist } q>0 \text { and } \delta>0 \text { such that } a(s) \geq s^{q}, s \in[0, \delta] .
\end{aligned}
$$

In the above $\mathrm{PDE}$ equation div and $\nabla$ denote the divergence and the gradient in $M$.

Received by the editors August 24, 2012.

2010 Mathematics Subject Classification. Primary 58J05; Secondary 35J92, 35J93. 
The asymptotic Dirichlet problem with boundary condition $\varphi \in C^{0}\left(\partial_{\infty} M\right)$ consists in finding a solution $u \in W_{\text {loc }}^{1, p}(M) \cap C^{0}(M)$ of $\mathcal{Q}=0$ in $M$ that extends continuously to $\partial_{\infty} M$ and $\left.u\right|_{\partial_{\infty} M}=\varphi$. By a solution $u$ of $\mathcal{Q}=0$ in $M$ we mean a weak solution, that is, $u$ is in the Sobolev space $W_{\text {loc }}^{1, p}(M)$ and satisfies

$$
\int_{M}\left\langle\frac{a(|\nabla u|)}{|\nabla u|} \nabla u, \nabla \xi\right\rangle=0
$$

for all all $\xi \in W_{0}^{1, p}(M)$.

In the Laplacian case this problem has been intensively investigated during the last three decades (see, for instance, And, C], $\mathrm{Hs}, \mathrm{Ne}, \mathrm{SY}$ and the references therein).

The notion of regularity of the boundary of a domain $\Omega \subset M$ is fundamental in elliptic PDE theory to prove that a solution of the Dirichlet problem of an elliptic PDE assumes a given value at the boundary of $\Omega$ (see GT]). To deal with the asymptotic Dirichlet problem in $M$ we extend this notion to the asymptotic boundary $\partial_{\infty} M$ of $M$ as follows.

We say that $M$ is regular at infinity with respect to $\mathcal{Q}$ if, given $C>0, x \in \partial_{\infty} M$, and a relatively open subset $W \subset \partial_{\infty} M$ containing $x$, there are an open subset $\Omega \subset$ $M$ such that $x \in \operatorname{Int}\left(\partial_{\infty} \Omega\right) \subset W$, and sub and supersolutions $\sigma, \Sigma \in C^{0}(M)$ of $\mathcal{Q}=$ 0 in $M$ (called barriers at $x$ ) such that $\sigma \leq 0 \leq \Sigma, \lim _{p \rightarrow x} \sigma(p)=\lim _{p \rightarrow x} \Sigma(p)=0$ and $\left.\sigma\right|_{M \backslash \Omega} \leq-C$ and $\left.\Sigma\right|_{M \backslash \Omega} \geq C$ (see Section 2 and Definition 2.1 for more details).

We prove that if $M$ is regular at infinity with respect to $\mathcal{Q}$ and if:

(a) there is an exhaustion of $M$ by an increasing sequence of $C^{\infty}$ bounded subdomains $\Omega_{k} \subset M$ such that the Dirichlet problem for $\mathcal{Q}=0$ is solvable in $\Omega_{k}$ for any boundary data which is in $C^{\infty}\left(\partial \Omega_{k}\right)$,

(b) sequences of solutions with uniformly bounded $C^{0}$ norm are compact in relatively compacts subsets of $M$,

then the asymptotic Dirichlet problem for $\mathcal{Q}$ is solvable for any continuous boundary data (Theorem 2.3).

In view of the classical and more recent results on geometric quasilinear elliptic PDE theory, conditions (a) and (b) hold in a large class of elliptic PDE's, thus reducing the solvability of this problem to the regularity of $M$ at infinity with respect to the operator $\mathcal{Q}$.

From the well known work of $\mathrm{H}$. Choi [C], it follows that if $M$ satisfies the convex conic neighborhood condition, namely, any two distinct points of $\partial_{\infty} M$ can be separated by $C^{2}$ convex open disjoint neighborhoods of these points in $\bar{M}$, then any point at infinity is regular for the Laplace operator (the 2-Laplacian); in particular, the asymptotic Dirichlet problem for the Laplace equation $\Delta u=0$ is solvable for any given continuous boundary data $\varphi$ at $\partial_{\infty} M$. Since Choi's proof using the convex conic neighborhood condition is heavily based on the linearity of the Laplacian operator, it does not apply directly to quasilinear elliptic PDE's, as the $p$-Laplacian or the minimal hypersurface PDE.

In the present work we propose a different notion of convexity; informally speaking, when one can extract from $\bar{M}$ a neighborhood of any point of $\partial_{\infty} M$ such that what remains is still convex (as it happens with strictly convex bounded domains). 
The precise definition is:

Definition 1.1. Let $M$ be a Hadamard manifold. We say that $M$ satisfies the strict convexity condition (SC condition) if, given $x \in \partial_{\infty} M$ and a relatively open subset $W \subset \partial_{\infty} M$ containing $x$, there exists a $C^{2}$ open subset $\Omega \subset \bar{M}$ such that $x \in \operatorname{Int}\left(\partial_{\infty} \Omega\right) \subset W$, where $\operatorname{Int}\left(\partial_{\infty} \Omega\right)$ denotes the interior of $\partial_{\infty} \Omega$ in $\partial_{\infty} M$, and $M \backslash \Omega$ is convex.

As we shall see, the SC condition gives an a priori control of the behavior at infinity of the solutions of divergence form quasilinear elliptic PDEs (1) subject to the conditions (2)-(4). In fact we prove that if $M$ satisfies the SC condition, then it is regular at infinity with respect to $\mathcal{Q}$ (Theorem 2.5). As a consequence, under the SC condition, when $\mathcal{Q}$ satisfies conditions (a) and (b), then the asymptotic Dirichlet problem for $\mathcal{Q}$ is solvable for any continuous boundary data (Theorem 2.6).

We observe that the minimal hypersurface PDE $\mathcal{M}=0$ and the $p$-Laplacian $\operatorname{PDE} \Delta_{p}=0, p>1$, are special cases of $\mathcal{Q}=0$, where $a(t)=t / \sqrt{1+t^{2}}$ and $a(t)=t^{p-1}$, respectively. It is easy to see that they both satisfy conditions (2), (3), (4) and, as we shall see later, both $\mathcal{M}$ and $\Delta_{p}$ satisfy conditions (a) and (b). Then, from Theorem 2.5. we obtain that if $M$ satisfies the SC condition, then the Dirichlet problem with prescribed continuous asymptotic data is solvable for $\mathcal{M}$ and $\Delta_{p}, p>1$ (Theorem 2.8).

To show the extent of the above results, we first prove that if $M$ has a rotationally symmetric metric, then it satisfies the SC condition (Theorem 3.4). By an adaptation of a nice construction due to Borbély [B1] we also prove that if $\inf _{B_{R+1}} K_{M} \geq-e^{2 k R} / R^{2+2 \epsilon}, R \geq R^{*}$, for some $R^{*}$ and $\epsilon>0$, where $B_{R+1}$ is the geodesic ball with radius $R+1$ centered at a fixed point of $M$, then our SC condition is satisfied (Theorem 3.5). Remark that 2-dimensional Hadamard manifolds (under the assumption $K_{M} \leq-k^{2}$ ) always satisfy the SC condition, since any two points of $\partial_{\infty} M$ can be connected by a geodesic.

The classes of Hadamard manifolds $M$ that we have considered essentially satisfy both Choi's convex conic condition and our SC condition. It may then be possible that these conditions are actually equivalent. So far, this remains an open problem. We also observe that if no convexity is required, then it was constructed by Ancona Anc and Borbély B2] examples of 3-dimensional Hadamard manifolds $M$ with $K_{M} \leq-1$ for which the asymptotic Dirichlet problem for the Laplace equation is not solvable for all continuous nonconstant boundary data. In [Ho] I. Holopainen extended Borbély's resulto to the $p$-Laplacian PDE, and, quite recently, the first author of the present article and I. Holopainen [HoR] extended these nonsolvability results to the class of PDEs (11) including, particularly, the minimal hypersurface PDE. We finally mention that the hypothesis $K_{M} \leq-k^{2}<0$ cannot be relaxed to $K_{M}<0$, even in dimension 2. This follows from the results of M. Rigoli and A. Setti in [RS] where the authors study the PDE (11) focusing on nonexistence Liouville type theorems (see Remark 2.7 below).

\section{The asymptotic Dirichlet PRoblem for Hadamard Manifolds WHICH SATISFY THE STRICT CONVEXITY CONDITION}

Let $M$ be a Hadamard manifold. We say that a function $\Sigma \in C^{0}(M)$ is a supersolution for $\mathcal{Q}$ if, given a bounded domain $U \subset M, u \in C^{0}(\bar{U})$ is a solution of $\mathcal{Q}=0$ in $U$, the condition $\left.u\right|_{\partial U} \leq\left.\Sigma\right|_{\partial U}$ implies that $u \leq\left.\Sigma\right|_{U}$. 
Given $x \in \partial_{\infty} M$ and an open subset $\Omega \subset M$ such that $x \in \partial_{\infty} \Omega$, an upper barrier for $\mathcal{Q}$ relative to $x$ and $\Omega$ with height $C$ is a function $\Sigma \in C^{0}(M)$ such that

(i) $\Sigma$ is a supersolution for $\mathcal{Q}$;

(ii) $\Sigma \geq 0$ and $\lim _{p \in M, p \rightarrow x} \Sigma(p)=0$, the limit with respect to the cone topology;

(iii) $\Sigma_{M \backslash \Omega} \geq C$.

Similarly, we define subsolutions and lower barriers.

Definition 2.1. Let $M$ be a Hadamard manifold. We say that $M$ is regular at infinity with respect to the differential operator $\mathcal{Q}$ if, given $C>0, x \in \partial_{\infty} M$ and an open subset $W \subset \partial_{\infty} M$ with $x \in W$, there exist an open set $\Omega \subset M$ such that $x \in \operatorname{Int} \partial_{\infty} \Omega \subset W$ and upper and lower barriers $\Sigma, \sigma: M \rightarrow \mathbb{R}$ relative to $x$ and $\Omega$, with height $C$.

Compare this definition with Definition 2.6 and Theorem 2.7 in C] for the case of the Laplace operator and also with Theorem 3.3 and Definition 3.4 in [HoV] for the case of the $p$-Laplacian.

The next lemma gives candidates to supersolutions of $\mathcal{Q}=0$, and hence to upper barriers. Although it is a well known result, we write the proof for the reader's convenience.

Lemma 2.2. Let $U \subset M$ be an open set and $v \in C^{0}(U) \cap W_{\text {loc }}^{1, p}(U)$ such that, for every $\xi \in C_{0}^{\infty}(U)$ with $\xi \geq 0$, it holds that

$$
\int_{U}\left\langle\frac{a(|\nabla v|}{|\nabla v|} \nabla v, \nabla \xi\right\rangle d x \geq 0
$$

( $v$ is frequently known as a weak supersolution of $\mathcal{Q}=0$ ). Then $v$ is a supersolution of $\mathcal{Q}=0$. In particular, if $v \in C^{2}(U)$ satisfies $\mathcal{Q}[v] \leq 0$, then $v$ is a supersolution.

Proof. We first notice that inequality (5) holds for all $0 \leq \xi \in C_{0}^{\infty}(U)$ if and only if it holds for all $\xi \in W_{0}^{1, p}(U)$, with $\xi \geq 0$ a.e.

In order to prove that $v$ is a supersolution, let $B \Subset U$ and $u \in C^{0}(\bar{B}) \cap W^{1, p}(B)$ such that $u \leq v$ on $\partial B$. We must prove that $u \leq v$ in $B$. For, notice that inequality (15) holds for the particular choice of $\xi$ given by $\xi=(u-v)_{+}:=\max \{u-v, 0\}$, by the remark above. On the other hand, since $u$ is a solution of $\mathcal{Q}=0$, it holds that

$$
\int_{B}\left\langle\frac{a(|\nabla u|}{|\nabla u|} \nabla u, \nabla \xi\right\rangle d x=0 .
$$

Therefore we may combine both expressions and obtain

$$
\int_{B}\left\langle\frac{a(|\nabla u|)}{|\nabla u|} \nabla u-\frac{a(|\nabla v|)}{|\nabla v|} \nabla v, \nabla u-\nabla v\right\rangle d x \leq 0 .
$$

On the other hand,

$$
\begin{array}{r}
\left\langle\frac{a(|\nabla u|)}{|\nabla u|} \nabla u-\frac{a(|\nabla v|)}{|\nabla v|} \nabla v, \nabla u-\nabla v\right\rangle=a(|\nabla u|)|\nabla u|^{2} \\
-\frac{a(|\nabla u|)}{|\nabla u|}\langle\nabla u, \nabla v\rangle-\frac{a(|\nabla v|)}{|\nabla v|}\langle\nabla u, \nabla v\rangle+a(|\nabla v|)|\nabla v| \\
\geq a(|\nabla u|)|\nabla u|-a(|\nabla u|)|\nabla v|-a(|\nabla v|)|\nabla u|+a(|\nabla v|)|\nabla v| \\
=(a(|\nabla u|)-a(|\nabla v|))(|\nabla u|-|\nabla v|),
\end{array}
$$


where the inequality is implied by Cauchy-Schwarz inequality; and since $a$ is increasing the last product must be $\geq 0$. Hence the integrated function on (6) is nonnegative, and since its integral is nonpositive, it vanish a.e. on $B$. Since $a$ is injective, it follows that $\nabla u=\nabla v$ a.e. in $B$ which implies that $(u-v)_{+}$is constant a.e. Since it is continuous, we conclude that it actually is constant in $B$, and since it vanishes on the boundary, $(u-v)_{+}=0$, which finishes the first part of the proof.

The second part is a consequence of integration by parts.

Theorem 2.3. Let $M$ be a Hadamard manifold with sectional curvature $K_{M} \leq$ $-k^{2}<0$ which is regular at infinity with respect to $\mathcal{Q}$. Assume moreover that

(a) there is a sequence of bounded $C^{\infty}$ domains $\Omega_{k} \subset M, k \in \mathbb{N}$, satisfying $\Omega_{k} \subset \Omega_{k+1}, \cup \Omega_{k}=M$, such that the Dirichlet problem for $\mathcal{Q}=0$ is solvable in $\Omega_{k}$ for any $C^{\infty}$ boundary data,

(b) sequences of solutions with uniformly bounded $C^{0}$ norm are compact in relatively compacts subsets of $M$.

Then the asymptotic Dirichlet problem of $\mathcal{Q}$ is solvable for any continuous boundary data.

Proof. Let $\phi \in C^{\infty}(M) \cap C^{0}(\bar{M})$ be a smooth extension of $\varphi$. Condition (a) allows us to solve the Dirichlet problem

$$
\left\{\begin{array}{l}
\mathcal{Q}[u]=0 \text { in } \Omega_{k}, u \in W_{\text {loc }}^{1, p}\left(\Omega_{k}\right) \cap C^{0}\left(\overline{\Omega_{k}}\right) \\
\left.u\right|_{\partial \Omega_{k}}=\phi
\end{array}\right.
$$

finding a solution $u_{k} \in C^{0}\left(\overline{\Omega_{k}}\right)$.

Condition (b) together with the diagonal method show that there exists a subsequence of $\left(u_{k}\right)$ (which we suppose to be $\left(u_{k}\right)$ ) converging uniformly on compact subsets of $M$ to a global solution of $\mathcal{Q}=0$, which we denote by $u$. One needs to show that $u$ extends continuously to $\partial_{\infty} M$ and satisfies $\left.u\right|_{\partial_{\infty} M}=\varphi$.

For, let $x \in \partial_{\infty} M$ and $\varepsilon>0$. Since $\varphi$ is continuous, there exists an open neighborhood $W \subset \partial_{\infty} M$ of $x$ such that $\varphi(y)<\varphi(x)+\varepsilon / 2$ for all $y \in W$. Furthermore, the regularity of $M$ at infinity with respect to $\mathcal{Q}$ implies that there exists an open subset $\Omega \subset M$ such that $x \in \operatorname{Int}\left(\partial_{\infty} \Omega\right) \subset W$ and $\Sigma: M \rightarrow \mathbb{R}$ upper barrier with respect to $x$ and $\Omega$ with height $C:=\max _{\bar{M}}|\phi|$.

Defining

$$
v(q):=\Sigma(q)+\varphi(x)+\varepsilon
$$

we claim that $u \leq v$ in $\Omega$.

Since $\phi$ is continuous, $\exists k_{0} \gg 0$ such that $\phi(q)<\varphi(x)+\varepsilon / 2$ for all $q \in \partial \Omega_{k} \cap \Omega$, $k \geq k_{0}$; we may choose $k_{0}$ such that $\Omega_{k_{0}} \cap \Omega \neq \emptyset$.

Set $V_{k}:=\Omega \cap \Omega_{k}, k \geq k_{0}$. Claim: $u_{k} \leq v$ in $V_{k}$. In fact, the inequality holds on $\partial V_{k}=\overline{\left(\partial \Omega_{k} \cap \Omega\right)} \cup \overline{\left(\partial \Omega \cap \Omega_{k}\right)}$ : on $\partial \Omega_{k} \cap \Omega$, it is true due to the choice of $k_{0}$; on $\partial \Omega \cap \Omega_{k}$, it holds because $\Sigma \geq \max |\varphi|$ on $\partial \Omega$, which implies that $\Sigma \geq u_{k}$, by the Comparison Principle (Lemma 2.2).

Also the Comparison Principle implies that $u_{k} \leq v$ in $V_{k}$; since it holds for all $k \geq k_{0}$, we have $u \leq v$ on $\Omega$.

It is also possible to define $v_{-}: M \rightarrow \mathbb{R}$ by $v_{-}(q):=\varphi(x)-\varepsilon-\Sigma(q)$ in order to obtain $u \geq v_{-}$in $\Omega$. It then holds that

$$
|u(q)-\varphi(x)|<\varepsilon+\Sigma(q), \forall q \in \Omega,
$$


and hence

$$
\limsup _{p \rightarrow x}|u(p)-\varphi(x)| \leq \varepsilon .
$$

The proof is complete, since $\varepsilon>0$ is arbitrary.

Remark 2.4. We remark that in the bounded case, the regularity of the domain seems to depend heavier on the operator. For instance, any bounded $C^{2}$ domain is regular for the Laplace equation as, for the mean curvature operator, the $C^{2}$ regularity is not enough, the domain has to be convex too.

Theorem 2.5. Let $M$ be a Hadamard manifold with sectional curvature $K_{M} \leq-k^{2}$ satisfying the $S C$ condition. Then $M$ is regular at infinity with respect to $\mathcal{Q}$.

Proof. Let $C>0$ and $x \in W \subset \partial_{\infty} M$ be given. Since $\mathcal{Q}[-u]=-\mathcal{Q}[u]$ it is enough to prove the existence of a barrier from above at $x$. Since $M$ satisfies the SC condition, there exists a $C^{2}$ open subset $\Omega$ of $M$ such that $x \in \operatorname{Int}\left(\partial_{\infty} \Omega\right) \subset W$ and such that $M \backslash \Omega$ is convex. Let $s: \Omega \rightarrow \mathbb{R}$ be the distance function to $\partial \Omega$. Since $M \backslash \Omega$ is convex and $K_{M} \leq-k^{2}$, we may apply comparison theorems (see Theorems 4.2 and 4.3 of [C] ) in order to obtain the estimative

$$
\Delta s \geq(n-1) k \tanh k s .
$$

On the other hand, since $a^{\prime}>0, a$ has an inverse function $a^{-1} \in C^{1}([0, \alpha))$ where $\alpha=\sup a \leq \infty$. Set $c=a(2 C)$.

We may then define a function $g:[0, \infty) \rightarrow \mathbb{R}, g \in C^{2}((0, \infty))$, possibly with $g(0)=\infty$, by

$$
g(s):=\int_{s}^{\infty} a^{-1}\left(c \cosh ^{1-n} k t\right) d t .
$$

We observe that, from the assumptions on $a$, the function $g$ is well defined. In fact, let $\tau$ satisfy

$$
c \cosh ^{1-n} k \tau=\delta .
$$

Assuming, without loss of generality, that $\delta<c$, such $\tau$ exists because $c \cosh ^{1-n} 0=$ $c$ and $\lim _{t \rightarrow+\infty} c \cosh ^{1-n} k t=0$. We have $a^{-1}(t) \leq t^{1 / q}$ if $a(t) \in[0, \delta]$, and therefore

$$
\begin{aligned}
g(s) & =\int_{s}^{\tau} a^{-1}\left(c \cosh ^{1-n} k t\right) d t+\int_{\tau}^{+\infty} a^{-1}\left(c \cosh ^{1-n} k t\right) d t \\
& \leq a^{-1}(c s)(\tau-s)+\int_{x}^{+\infty}\left(c \cosh ^{1-n} k t\right)^{\frac{1}{q}} d t \\
& \leq a^{-1}(c s) \tau+(2 c)^{\frac{1}{q}} \int_{\tau}^{+\infty} e^{-\frac{k t}{q}} d t=a^{-1}(c s) \tau+\frac{(2 c)^{\frac{k}{q}}}{q} e^{-k \tau}<+\infty
\end{aligned}
$$

for all $s>0$. Furthermore,

$$
g(0)>\int_{0}^{1} a^{-1}\left(c \cosh ^{1-n} k t\right) d t \geq a^{-1}(c)=2 C
$$

and $\lim _{s \rightarrow \infty} g(s)=0$. Therefore we may define $v: \Omega \rightarrow \mathbb{R}$ as

$$
v(p):=g(s(p)),
$$

and we want to show that $\mathcal{Q}(v) \leq 0$. Notice that

$$
\nabla v(p)=g^{\prime}(s(p)) \nabla s(p)=-a^{-1}\left(c \cosh ^{1-n} k s(p)\right) \nabla s
$$


and then $|\nabla v|=\left|g^{\prime}(s(p))\right|=a^{-1}\left(c \cosh ^{1-n} k s(p)\right)$; furthermore, it holds that $\nabla v /|\nabla v|=-\nabla s$. Combining the previous expressions, we obtain

$$
\begin{aligned}
\mathcal{Q}(v)= & \operatorname{div}\left(a\left(\left|g^{\prime}(s(p))\right| \nabla s(p)\right)\right) \\
= & \operatorname{div}\left(a\left(-a^{-1}\left(c \cosh ^{1-n} k s(p)\right)\right) \nabla s(p)\right) \\
= & \operatorname{div}\left(-c \cosh ^{1-n} k s(p) \nabla s(p)\right) \\
= & -(1-n) c k \cosh ^{-n} k s(p) \sinh k s(p)\langle\nabla s(p), \nabla s(p)\rangle \\
& -c \cosh ^{1-n} k s(p) \Delta s(p) \\
\leq & (n-1) c k \cosh ^{-n} k s(p) \sinh k s(p) \\
& -(n-1) c \cosh ^{1-n} k s(p) k \tanh k s(p)=0,
\end{aligned}
$$

and hence, by Lemma 2.2, $v$ is a supersolution on $\Omega$.

To finish with the proof, define the global supersolution $\Sigma \in C^{0}(\bar{M})$ by

$$
\Sigma(p)= \begin{cases}\min \{v(p), C\} & \text { if } p \in \Omega \\ C & \text { if } p \in \bar{M} \backslash \Omega,\end{cases}
$$

which is of course an upper barrier relative to $x$ and $\Omega$ with height $C$.

As a consequence of Theorems 2.3 and 2.5 we obtain

Theorem 2.6. Let $M$ be a Hadamard manifold with sectional curvature satisfying $K_{M} \leq-k^{2}$. Assume that $M$ satisfies the $S C$ condition and that $\mathcal{Q}$ satisfies conditions (a) and (b). Then the asymptotic Dirichlet problem of $\mathcal{Q}$ is solvable for any continuous boundary data.

Remark 2.7. Since in the two-dimensional case the hypothesis $K_{M} \leq k^{2}<0$ implies the $\mathrm{SC}$ condition, it is interesting to know if Theorem 2.6 remains true if we just require $K_{M}<0$. We may see that if

$$
0>\inf _{\partial B_{r}} K_{M} \geq-\frac{1}{r^{2} \log r}
$$

where $B_{r}$ is a geodesic ball centered at some fixed point of $M$, for $r \geq r_{0}, r_{0}$ large enough, then the Bishof comparison theorem implies that $\left|\partial B_{r}\right| \leq r \log r$, where $\left|\partial B_{r}\right|$ is the volume of $\partial B_{r}$. It follows that for $p \geq 2$ and $s$ large enough we have

$$
\int_{s}^{\infty} \frac{d r}{\left|\partial B_{r}\right|^{\frac{1}{p-1}}} \geq \int_{s}^{\infty} \frac{d r}{r \log r}=\infty .
$$

From Theorem A of [RS] it follows that there is no entire bounded solution of $\mathcal{Q}=0$ in $M$ besides the constant functions whenever the function $a$ that defines $\mathcal{Q}$ satisfies $a(t) \leq C t$ near the origin for some $C>0$. This is the case, for instance, for the $p$-Laplacian, $p \geq 2$, and minimal PDEs. In particular, the asymptotic Dirichlet problem for those cases is not solvable for any continuous boundary data in $\partial_{\infty} M$ if $K_{M}$ satisfies (9). Hence, Theorem [2.6 is false if one replaces the hypothesis $K_{M} \leq-k^{2}<0$ by $K_{M}<0$. 
The nonexistence condition (9) for the curvature is sharp for the Laplacian and the minimal surface PDEs in the sense that if

$$
\sup _{\partial B_{r}} K_{M} \leq-\frac{1+\varepsilon}{r^{2} \log r}(\operatorname{dim} M=2)
$$

for some $\varepsilon>0$, then it was proved by R. W. Neel in Ne that the asymptotic Dirichlet problem for the Laplacian PDE is solvable for any continuous boundary data. In RT it is proved that the same hypothesis (10) on the curvature of a rotationally symmetric $M$ also implies the solvability of the minimal surface equation in $M$ for any prescribed continuous boundary data. We note that these remarks in the case of the minimal PDE give a partial answer to a question formulated in GR.

Theorem 2.8. Let $M$ be a Hadamard manifold with sectional curvature satisfying $K_{M} \leq-k^{2}$ and assume that $M$ satisfies the $S C$ condition. Then the asymptotic Dirichlet problems for the minimal hypersurface $\mathcal{M}=0$ PDE and the $p$-Laplacian $\Delta_{p}=0 P D E, p>1$, are uniquely solvable for any given continuous boundary data at infinity. The solution is in the classical sense in the minimal case and in the weak sense in the p-Laplacian case. Precisely: Given $\varphi \in C^{0}\left(\partial_{\infty} M\right)$ there are solutions $u \in C^{\infty}(M) \cap C^{0}(\bar{M})$ of $\mathcal{M}[u]=0$ and $v \in C^{1, \alpha}(M) \cap C^{0}(\bar{M})$ of $\Delta_{p}[v]=0$ such that $\left.u\right|_{\partial_{\infty} M}=\left.v\right|_{\partial_{\infty} M}=\varphi$.

Proof. Due to Theorem 2.5 above one only has to prove that conditions (a) and (b) are satisfied for $\mathcal{M}$ and $\Delta_{p}$. Fixing a point $o \in M$ we take $\Omega_{k}:=B_{k}$, the geodesic ball of $M$ with radius $k$ centered at $o$. Choose $k$ and let $\psi \in C^{\infty}\left(\partial B_{k}\right)$ be given. In the $p$-Laplacian case, the same arguments used for proving the existence of solutions of the $p$-Laplacian PDE in $\mathbb{R}^{n}$ with prescribed $C^{\infty}$ boundary data apply to prove the existence of a weak solution $u_{k} \in C^{1, \alpha}\left(\overline{B_{k}}\right)$ of $\Delta_{p}=0$ in $B_{k}$ such that $\left.u_{k}\right|_{\partial B_{k}}=\psi$ for some $\alpha \in(0,1)$ which depends only on $p$. This proves that condition (a) is satisfied for $\Delta_{p}$. The interior gradient estimates of [ $\left.\mathrm{KN}\right]$ and standard elliptic PDE theory implies that uniformly $C^{0}$ bounded sequences of solutions of $\Delta_{p}=0$ are compact on relatively compact subsets of $M$ proving that condition (b) is satisfied for $\Delta_{p}$. In the case of the minimal PDE, condition (a) follows from Theorem 1 of DHL and elliptic regularity theory. Condition (b) follows from Theorem 1.1 of [S] and standard arguments from PDE elliptic theory. This proves the theorem.

\section{Applications}

In this section, we first show that rotationally symmetric manifolds with sectional curvature $K_{M} \leq-k^{2}$ satisfy the strict convexity condition. The proof of this fact is constructive, and there is almost no difference between the 3-dimensional and the $n$-dimensional case, $n \geq 3$, as we shall see in the final constructions. The existence results ensured by Theorem 2.8 in rotationally manifolds, for the minimal and $p$-Laplacian PDE's, $p>1$, have already been obtained by different authors in different papers, some of them with weaker conditions on the sectional curvature (see [GR], EFR] and [V]).

Let $M$ be an $(n+1)$-dimensional rotationally symmetric Hadamard manifold with center $o$. We may treat $M$ as the set

$$
\mathbb{R}^{n+1}=\left\{\left(x_{1}, \ldots, x_{n+1}\right) \mid x_{i} \in \mathbb{R}\right\}
$$


parametrized by $r$, the distance to $(0, \ldots, 0)=o$, and $\theta_{1}, \ldots, \theta_{n}$, with

$$
\begin{aligned}
& x_{1}=r \sin \theta_{1} \sin \theta_{2} \ldots \sin \theta_{n}, \\
& x_{2}=r \cos \theta_{1} \sin \theta_{2} \ldots \sin \theta_{n}, \\
& x_{3}=r \cos \theta_{2} \sin \theta_{3} \ldots \sin \theta_{n}, \\
& \ldots \\
& x_{n}=r \cos \theta_{n-1} \sin \theta_{n}, \\
& x_{n+1}=r \cos \theta_{n},
\end{aligned}
$$

endowed with the metric

$$
d r^{2}+f(r)^{2} d \omega^{2}
$$

where $d \omega^{2}$ is the standard metric of $\mathbb{S}^{n}$. We use the notation $M=\left(\mathbb{R}^{n+1}, f\right)$ for $M$ if it is rotationally symmetric with metric given by (12).

Remark 3.1. Notice that:

(i) The angles at the origin $o$ are the same whatever is the function $f$ that gives the metric.

(ii) The parameter $\theta_{n}$ is the angle with the direction $(0, \ldots, 0,1)$.

The vector fields defined by

$$
U:=\frac{\partial}{\partial r}, V_{i}:=\frac{\partial}{\partial \theta_{i}}
$$

give a local frame for $M \backslash\{o\}$. If we use the notation $g_{00}:=\langle U, U\rangle$ and $g_{i i}:=\left\langle V_{i}, V_{i}\right\rangle$, it is easy to see that

$$
\begin{aligned}
& g_{i i}=f(r)^{2} \sin ^{2} \theta_{i+1} \sin ^{2} \theta_{i+2} \ldots \sin ^{2} \theta_{n}, 1 \leq i \leq n-1, \\
& g_{00}=1, g_{n n}=f(r)^{2} \text { and } g_{i j}=0 \text { if } i \neq j .
\end{aligned}
$$

We now fix $\gamma:[0,+\infty) \rightarrow M$ a unit speed geodesic ray with $\gamma(0)=o$. Since any geodesic ray with the same properties may be mapped by an isometry on $\gamma$, we may assume without loss of generality that $\gamma(s)=(0, \ldots, 0, s), s \geq 0$.

Let $r(t), \theta(t)$ be given by the solution of the system

$$
\left\{\begin{aligned}
r^{\prime}(t) & =\cosh k R \sin \theta(t), \\
\theta^{\prime}(t) & =\frac{k \sinh k R}{\sinh ^{2}(k r(t))} \\
r(0) & =R, \theta(0)=0
\end{aligned}\right.
$$

and let $S_{R} \subset \mathbb{R}^{n+1}$ be the hypersurface parametrized by

$$
\begin{aligned}
\left(t, \theta_{1}, \ldots, \theta_{n-1}\right) \mapsto & \left(r(t) \sin \theta_{1} \sin \theta_{2} \ldots \sin \theta_{n-1} \sin \theta(t),\right. \\
& r(t) \cos \theta_{1} \sin \theta_{2} \ldots \sin \theta_{n-1} \sin \theta(t), \\
& r(t) \cos \theta_{2} \sin \theta_{3} \ldots \sin \theta_{n-1} \sin \theta(t), \\
& \left.\ldots, r(t) \cos \theta_{n-1} \sin \theta(t), r(t) \cos \theta(t)\right),
\end{aligned}
$$

where $r(t)$ and $\theta(t)$ are given by (15).

Lemma 3.2. If $M=\left(\mathbb{R}^{n+1}, \sinh (k r) / k\right)$, i.e., $M$ is the hyperbolic space with constant sectional curvature equal to $-k^{2}$, then the hypersurface $S_{R}$ is the hyperbolic 
totally geodesic hypersurface that intersects $\gamma$ orthogonally at $(0, \ldots, 0, R)$. Moreover,

$$
\partial_{\infty} S_{R}=\left\{\tilde{\gamma}(\infty) \mid \tilde{\gamma}(0)=o, \varangle_{o}\left(\tilde{\gamma}^{\prime}(0), \gamma^{\prime}(0)\right)=\arccos (\tanh k R)\right\} .
$$

The preceding lemma (that will be proved after the next proposition) shows that $S_{R}$ exists and divides $\mathbb{R}^{n+1}$ in two connected components. These are both convex if $\mathbb{R}^{n+1}$ is endowed with the hyperbolic metric, since the second fundamental form of $S_{R}$ vanishes. The next proposition shows that $S_{R}$ has the desired properties if we replace the hyperbolic metric by a rotationally symmetric one, if $K_{M} \leq-k^{2}$.

We assume that $R>0$, and we denote by $\Omega^{\prime}$ the connected component of $\mathbb{R}^{n+1} \backslash S_{R}$ that contains the point $(0,0, \ldots, 0)$.

Lemma 3.3. Let $S_{R} \subset \mathbb{R}^{n+1}$ be the hypersurface defined as above, with $R>0$. If $M=\left(\mathbb{R}^{n+1}, f\right)$ has sectional curvature $K_{M}$ satisfying $K_{M} \leq-k^{2}$, then $\Omega^{\prime}$ is convex.

Proof. Since $S_{R}$ is parametrized by (16), the vector fields defined by

$$
T:=\frac{\partial}{\partial t}, V_{1}, V_{2}, \ldots, V_{n-1}
$$

give an orthogonal frame of $T_{p} S_{R}$ for any $p \in S_{R}$. Furthermore, notice that $T=$ $r^{\prime}(t) U+\theta^{\prime}(t) V_{n}$.

It is a straightforward calculation to verify that

$$
N\left(t, \theta_{1}, \ldots, \theta_{n-1}\right):=-f(r(t)) \theta^{\prime}(t) U+\frac{r^{\prime}(t)}{f(r(t))} V_{n}
$$

is normal to $S_{R}$ pointing to $\Omega^{\prime}$. In order to show that $\Omega^{\prime}$ is convex, we use Theorem 4.1 of [C]: It suffices to prove that the second fundamental form of $S_{R}$ with respect to $N$ is nonnegative. It is then sufficient to show that

$$
\left\langle\nabla_{T} T, N\right\rangle,\left\langle\nabla_{V_{i}} V_{i}, N\right\rangle \geq 0, \forall i \in\{1, \ldots, n-1\},
$$

where $\nabla$ is the Levi-Civita connection of $\left(\mathbb{R}^{n+1}, f\right)$.

We start with $\left\langle\nabla_{T} T, N\right\rangle$ :

$$
\begin{aligned}
\left\langle\nabla_{T} T, N\right\rangle & =-f \theta^{\prime}\left\langle\nabla_{T} T, U\right\rangle+\frac{r^{\prime}}{f}\left\langle\nabla_{T} T, V\right\rangle \\
& =-f \theta^{\prime} T\langle T, U\rangle+f \theta^{\prime}\left\langle T, \nabla_{T} U\right\rangle+\frac{r^{\prime}}{f} T\langle T, V\rangle-\frac{r^{\prime}}{f}\left\langle T, \nabla_{T} V\right\rangle \\
& =-f \theta^{\prime} r^{\prime \prime}+f \theta^{\prime}\left\langle T, \nabla_{T} U\right\rangle+\frac{r^{\prime}}{f}\left(f^{2} \theta^{\prime}\right)^{\prime}-\frac{r^{\prime}}{f}\left\langle T, \nabla_{T} V\right\rangle .
\end{aligned}
$$

Notice that

$$
\begin{aligned}
\left\langle T, \nabla_{T} U\right\rangle & =r^{\prime 2}\left\langle U, \nabla_{U} U\right\rangle+r^{\prime} \theta^{\prime}\left(\left\langle U, \nabla_{V} U\right\rangle+\left\langle V, \nabla_{U} U\right\rangle\right)+\theta^{\prime 2}\left\langle V, \nabla_{V} U\right\rangle \\
& =0+0+0+\theta^{\prime 2} \frac{1}{2} U\left(\|V\|^{2}\right)=\theta^{\prime 2} f f_{r}
\end{aligned}
$$

and

$$
\begin{aligned}
\left\langle T, \nabla_{T} V\right\rangle & =r^{\prime 2}\left\langle U, \nabla_{U} V\right\rangle+r^{\prime} \theta^{\prime}\left(\left\langle U, \nabla_{V} V\right\rangle+\left\langle V, \nabla_{U} V\right\rangle\right)+\theta^{\prime 2}\left\langle V, \nabla_{V} V\right\rangle \\
& =r^{\prime} \theta^{\prime}\left(-\frac{1}{2} U\left(\|V\|^{2}\right)+\frac{1}{2} U\left(\|V\|^{2}\right)\right)+\theta^{\prime 2} \frac{1}{2} V\left(\|V\|^{2}\right)=0 .
\end{aligned}
$$


Then, replacing (20) and (21) on (19), it follows that

$$
\left\langle\nabla_{T} T, N\right\rangle=f \theta^{\prime}\left(f f_{r} \theta^{2}-r^{\prime \prime}\right)+\frac{r^{\prime}}{f}\left(f^{2} \theta^{\prime}\right)^{\prime}
$$

We now compute $\left\langle\nabla_{V_{i}} V_{i}, N\right\rangle$ :

$$
\begin{aligned}
\left\langle\nabla_{V_{i}} V_{i}, N\right\rangle & =-f \theta^{\prime}\left\langle\nabla_{V_{i}} V_{i}, U\right\rangle+\frac{r^{\prime}}{f}\left\langle\nabla_{V_{i}} V_{i}, V\right\rangle \\
& =f \theta^{\prime}\left\langle V_{i}, \nabla_{V_{i}} U\right\rangle-\frac{r^{\prime}}{f}\left\langle V_{i}, \nabla_{V_{i}} V\right\rangle \\
& =f \theta^{\prime}\left\langle V_{i}, \nabla_{U} V_{i}\right\rangle-\frac{r^{\prime}}{f}\left\langle V_{i}, \nabla_{V} V_{i}\right\rangle \\
& =f \theta^{\prime} \frac{1}{2} U\left(\left\|V_{i}\right\|^{2}\right)-\frac{r^{\prime}}{f} \frac{1}{2} V\left(\left\|V_{i}\right\|^{2}\right) \\
& =f^{2} f_{r} \theta^{\prime 2} \theta_{i+1} \ldots \sin ^{2} \theta_{n}-r^{\prime 2} \theta_{i+1} \sin ^{2} \theta_{n-1} \sin \theta_{n} \cos \theta_{n} \\
& =f^{2} \sin ^{2} \theta_{i+1} \ldots \sin ^{2} \theta_{n}\left(f_{r} \theta^{\prime}-\frac{r^{\prime}}{f} \cot \theta_{n}\right) .
\end{aligned}
$$

On the other hand, since $r$ and $\theta$ satisfy (15), we have $\cos \theta=\tanh k R \operatorname{coth} k r$, and then

$$
\begin{aligned}
f f_{r} \theta^{\prime 2}-r^{\prime \prime} & =f f_{r} \theta^{\prime 2}-\theta^{\prime} \cosh k R \cos \theta \\
& =\theta^{\prime}\left(f f_{r} \frac{k \sinh k R}{\sinh ^{2} k r}-\cosh k R \operatorname{coth} k r \tanh k R\right) \\
& =\theta^{\prime}\left(f f_{r} \frac{k \sinh k R}{\sinh ^{2} k r}-\sinh k R \frac{\cosh k r}{\sinh k r}\right) \\
& =\frac{\theta^{\prime}}{\sinh ^{2} k r} k \sinh k R\left(f f_{r}-\cosh k r \frac{\sinh k r}{k}\right) \geq 0,
\end{aligned}
$$

where the last inequality is true by Lemma 3.7 (see Section 3.1 below). Furthermore, $\theta^{\prime \prime}=-2 r^{\prime} \theta^{\prime} k \operatorname{coth} k r$, and then

$$
\begin{aligned}
r^{\prime}\left(f^{2} \theta^{\prime}\right)^{\prime} & =r^{\prime}\left(2 f f_{r} r^{\prime} \theta^{\prime}+f^{2} \theta^{\prime \prime}\right) \\
& =2 r^{\prime 2} \theta^{\prime}\left(f f_{r}-f^{2} k \operatorname{coth} k r\right)=2 r^{\prime 2} \theta^{\prime} f^{2}\left(\frac{f_{r}}{f}-k \operatorname{coth} k r\right) \geq 0,
\end{aligned}
$$

where the last inequality is again consequence of Lemma 3.7. Hence $\left\langle\nabla_{T} T, N\right\rangle \geq 0$.

Moreover,

$$
\begin{aligned}
f_{r} \theta^{\prime}-\frac{r^{\prime}}{f} \cot \theta & =k \sinh k R \frac{f_{r}}{\sinh ^{2} k r}-\cosh k R \frac{\cos \theta}{f} \\
& =\sinh k R\left(k \frac{f_{r}}{\sinh ^{2} r}-\frac{\operatorname{coth} k r}{f}\right) \\
& =\frac{k \sinh k R}{f \sinh ^{2} k r}\left(f f_{r}-\cosh k r \frac{\sinh k r}{k}\right) \geq 0,
\end{aligned}
$$

where the last inequality again comes from $f(r) \geq(\sinh k r) / k$ and $f^{\prime}(r) \geq \cosh k r$. Therefore $\left\langle\nabla_{V_{i}} V_{i}, N\right\rangle \geq 0$ for all $i \in\{1, \ldots, n-1\}$, and then $\Omega^{\prime}$ is convex. 
Proof of Lemma 3.2. In the hyperbolic space we have $f(r)=(\sinh k r) / k$. If we replace $f(r)$ by $(\sinh k r) / k$ on the computation done on the proof of the preceding proposition, it is easy to see that $\left\langle\nabla_{T} T, N\right\rangle=0=\left\langle\nabla_{V_{i}} V_{i}, N\right\rangle$. To conclude, notice that $\cos \theta(t)=\tanh k R \operatorname{coth} k r(t)$ implies that

$$
\lim _{t \rightarrow+\infty} \cos \theta(t)=\tanh k R, \lim _{t \rightarrow-\infty} \cos \theta(t)=-\tanh k R,
$$

which implies that

$$
\partial_{\infty} S_{R}=\left\{\tilde{\gamma}(\infty) \mid \tilde{\gamma}(0)=o, \varangle_{o}\left(\tilde{\gamma}^{\prime}(0), \gamma^{\prime}(0)\right)=\arccos (\tanh k R)\right\} .
$$

Theorem 3.4. If $M$ is a rotationally symmetric Hadamard manifold with $K_{M} \leq$ $-k^{2}$, then $M$ satisfies the $S C$ condition.

Proof. Let $x \in \partial_{\infty} M$ and let $W \subset \partial_{\infty} M$ be a relatively open subset such that $x \in$ Int $W$. Let $\gamma:[0,+\infty) \rightarrow M$ be the geodesic unit speed ray such that $\gamma(0)=o$ and $\gamma(\infty)=x$ and let $\alpha \in(0, \pi / 2)$ be such that

$$
\left\{\widetilde{\gamma}(\infty) \mid \widetilde{\gamma}(0)=o, \varangle\left(\widetilde{\gamma}^{\prime}(0), \gamma^{\prime}(0)\right) \leq \alpha\right\} \subseteq W .
$$

Choose $R \gg 0$ in such a way that $\tanh k R=\cos \alpha$ and construct $S_{R}$ e $\Omega^{\prime}$ as above. Setting $\Omega:=M \backslash \overline{\Omega^{\prime}}$, Lemma 3.3 gives us that $M \backslash \Omega$ is convex and furthermore

$$
\partial_{\infty} \Omega=\left\{\widetilde{\gamma}(\infty) \mid \widetilde{\gamma}(0)=o, \varangle\left(\widetilde{\gamma}^{\prime}(0), \gamma^{\prime}(0)\right) \leq \alpha\right\}
$$

since the angles at the origin $o$ do not depend on the function $f$ that defines the metric, and hence $\partial_{\infty} S_{R}$ is given by (17). It follows that $\Omega$ satisfies all desired conditions, which concludes the proof.

We now consider the case that $M$ is a general Hadamard manifold with $K_{M} \leq$ $-k^{2}, k>0$, with a control on the decay of the sectional curvature. We prove:

Theorem 3.5. Let $k>0$ be given. Suppose that $K_{M} \leq-k^{2}$ and that there exists $o \in M$ such that

$$
K_{R}:=\min \left\{K_{M}(\Pi) ; \Pi \text { is a } 2 \text {-plane in } T_{p} M, p \in B_{R+1}(o)\right\}
$$

satisfies

$$
K_{R} \geq-\frac{e^{2 k R}}{R^{2+2 \epsilon}}, R \geq R^{*},
$$

for some constants $\epsilon, R^{*}>0$. Then, given $\gamma(\infty) \in \partial_{\infty} M$ with $\gamma(0)=o$ and $0<\alpha<\pi / 2$, there exists a convex set $C \subset M$ such that

(i) $\partial_{\infty} C \supseteq\left\{\tilde{\gamma}(\infty) \mid \varangle_{o}(\tilde{\gamma}(\infty), \gamma(\infty)) \geq 2 \alpha\right\}$,

(ii) $C \cap T\left(\gamma^{\prime}(0), \alpha, r_{0}+1\right)=\emptyset$ for some $r_{0} \geq R^{*}$ large enough.

In particular, $M$ satisfies the $S C$ condition.

We observe that it is easy to check that if (25) holds for some point $o$, then it holds for all points, changing the constant $R^{*}$ if necessary.

For the proof of Theorem 3.5 we follow the construction of Anderson And and Borbély B1]. Both results are concerned with the existence of manifolds that satisfy the convex conic neighborhood condition of Choi. Since we are interested in a similar but more distinct result than Anderson and Borbély, we need to rewrite the proof with some adaptations. The principal idea is that since the sectional curvature is bounded from above by $-k^{2}, k>0$, all geodesic spheres have principal 
curvatures greater than $k$ (if we orient them inwards). It allows us to take out a small piece of the geodesic spheres and with the remaining set still convex. We observe that Borbély requires a somewhat stronger condition, namely: $\inf _{B_{R}} K_{M} \geq$ $-e^{\lambda R}, R \geq R^{*}$, for some $R^{*}$ and $0<\lambda<1 / 3$.

We observe that I. Holopainen and A. Vähäkangas in $[\mathrm{HoV}$ prove that the $p$-Laplacian is regular at infinity under the same hypothesis on the curvature of $M$, namely, $K_{M} \leq-k^{2}$ and condition (25) (see Theorem 3.21 and Corollary 3.23 of [HoV] $)$. It is interesting to note that the authors in [HoV], using a quite different technique, arrived at Corollary 3.23 requiring, as we did, the same decay condition for the sectional curvature of $M$.

We start with an arbitrary choice of a smooth function $\phi:[0,+\infty) \rightarrow \mathbb{R}$ such that $0 \leq \phi \leq 1, \phi([0,1 / 2])=0, \phi \equiv 1$ on $[1,+\infty)$ and $\phi^{\prime} \geq 0$. Let $L>0$ be such that $\phi^{\prime}, \phi^{\prime \prime} \leq L$. For $p \in S_{R}(o)$, let $f_{p}: M \rightarrow \mathbb{R}$ be given by

$$
f_{p}(x)=\phi\left(\rho_{p}(x)\right)
$$

where $\rho_{q}$ denotes the distance function to $q \in M$.

From now on, we use the notation $a_{R}:=\sqrt{-K_{R}}$.

Lemma 3.6. There exists $\beta>0$ which depends only on $L$ and $k$ such that if

$$
\varepsilon_{R}:=\beta R^{1+\epsilon} e^{-k R},
$$

then the sublevel set

$$
\left\{x \in M ;\left(\rho_{o}-\varepsilon_{R} f_{p}\right)(x) \leq R\right\}
$$

is a strictly convex set for all $R \geq \max \left\{1, R^{*}\right\}$ and $p \in S_{R}(o)$.

Proof. It suffices to prove that the Hessian of $\rho_{o}-\varepsilon_{R} f_{p}$ is positive-definite for all $X \perp \nabla\left(\rho_{o}-\varepsilon_{R} f_{p}\right)$. Let $X \perp \nabla\left(\rho_{o}-\varepsilon_{R} f_{p}\right)$. Then

$$
\begin{aligned}
& D^{2}\left(\rho_{o}-\varepsilon_{R} f_{p}\right)(X, X)=\left\langle\nabla_{X}\left(\nabla \rho_{o}-\varepsilon_{R} \phi^{\prime} \nabla \rho_{p}\right), X\right\rangle \\
= & D^{2} \rho_{o}(X, X)-\varepsilon_{R} \phi^{\prime} D^{2} \rho_{p}(X, X)-\varepsilon_{R} \phi^{\prime \prime}\left\langle\nabla \rho_{p}, X\right\rangle^{2} .
\end{aligned}
$$

Since $\rho_{o}$ is a distance function, we have

$$
D^{2} \rho_{o}(X, X)=D^{2} \rho_{o}\left(X^{\perp}, X^{\perp}\right),
$$

where $X^{\perp}=X-\left\langle X, \nabla \rho_{o}\right\rangle \nabla \rho_{o}$; furthermore, $\left\langle X, \nabla \rho_{o}\right\rangle=\varepsilon_{R} \phi^{\prime}\left\langle X, \nabla \rho_{p}\right\rangle$. Finally, since $K_{M} \leq-k^{2}$, the Hessian Comparison Theorem implies the estimative $D^{2} \rho_{o}\left(X^{\perp}, X^{\perp}\right) \geq k\left\|X^{\perp}\right\|^{2}$, and therefore

$$
\begin{aligned}
D^{2} \rho_{o}(X, X) & \geq k\left(\|X\|^{2}-\left\langle X, \nabla \rho_{o}\right\rangle^{2}\right) \\
& =k\left(\|X\|^{2}-\varepsilon_{R} \phi^{\prime}\left\langle X, \nabla \rho_{p}\right\rangle\left\langle X, \nabla \rho_{o}\right\rangle\right) \geq k\left(1-\varepsilon_{R} L\right)\|X\|^{2} .
\end{aligned}
$$

On the other hand, since $\phi^{\prime} \equiv 0$ on $[1,+\infty)$ and $K_{M} \geq-a_{R}^{2}$ in $B_{R+1}(o)$, it is possible to apply the Hessian Comparison Theorem in the other direction to obtain

$$
\begin{aligned}
\phi^{\prime} D^{2} \rho_{p}(X, X) & \leq \phi^{\prime} a_{R} \operatorname{coth}\left(a_{R} \rho_{p}\right)\left\|X-\left\langle X, \nabla \rho_{p}\right\rangle \nabla \rho_{p}\right\|^{2} \\
& =\phi^{\prime} a_{R} \operatorname{coth}\left(a_{R} \rho_{p}\right)\left(\|X\|^{2}-\left\langle X, \nabla_{p}\right\rangle^{2}\right) \\
& \leq \phi^{\prime} a_{R} \operatorname{coth}\left(a_{R} \rho_{p}\right)\|X\|^{2} .
\end{aligned}
$$

Since we have $\phi^{\prime} \equiv 0$ on $[0,1 / 2]$, with $a_{R} \geq 1$, we also obtain

$$
\phi^{\prime} D^{2} \rho_{p}(X, X) \leq \phi^{\prime} a_{R} \operatorname{coth}\left(a_{R} / 2\right)\|X\|^{2} \leq L a_{R} \operatorname{coth}(k / 2)\|X\|^{2} .
$$


Therefore, if $\|X\|=1$, using that $a_{R} \leq e^{k R} R^{-(1+\epsilon)}$, it holds that

$$
\begin{array}{r}
D^{2}\left(\rho_{o}-\varepsilon_{R} f_{p}\right)(X, X) \geq k\left(1-\varepsilon_{R} L\right)-\varepsilon_{R} L a_{R} \operatorname{coth}(k / 2)-\varepsilon_{R} L \\
=k\left(1-\beta e^{-k R} R^{1+\epsilon} L\right)-\beta e^{-k R} R^{1+\epsilon} L\left(a_{R} \operatorname{coth}(k / 2)-1\right) \\
\geq k-\beta(m(k+1)+\operatorname{coth}(k / 2)) L,
\end{array}
$$

where $m:=\max \left\{e^{-k R} R^{1+\epsilon} \mid R \geq 1\right\}$, and then, if we choose

$$
\beta:=\frac{k}{2 L(m(k+1)+\operatorname{coth}(k / 2))},
$$

we obtain $D^{2}\left(\rho_{o}-\varepsilon_{R} f_{p}\right)(X, X) \geq k / 2$, which concludes the proof.

Proof of Theorem 3.5. We start with $r_{0} \geq R^{*}$ to be determined later, and let $v:=$ $\gamma^{\prime}(0) \in T_{o} M$. Define the following sets:

$$
\left.C_{0}:=B_{r_{0}}, \quad T_{0}:=\left\{p \in S_{r_{0}} ; \varangle\left(\gamma_{o p}, v\right)\right)<\alpha\right\}, \quad D_{0}=\emptyset .
$$

Due to Lemma 3.6. there exists a uniform $\varepsilon_{0}:=\varepsilon_{r_{0}}$ such that for each $p \in S_{r_{0}}(o)$, the sublevel set

$$
C_{1, p}:=\left\{x \in M ;\left(\rho_{o}-\varepsilon_{0} f_{p}\right)(x) \leq r_{0}\right\}
$$

is a convex set. We therefore define the second collection of sets as follows:

$$
\begin{aligned}
& \widetilde{C_{1}}:=\bigcap_{p \in T_{0}} C_{1, p}, \quad C_{1}:=\widetilde{C_{1}} \backslash D_{0}, \quad r_{1}:=r_{0}+\varepsilon_{0}, \\
& D_{1}:=B_{r_{1}}(0) \backslash C_{1}, \quad T_{1}:=\overline{S_{r_{1}}(o) \backslash \partial C_{1}} .
\end{aligned}
$$

Notice that $C_{1}$ is convex, since it is the intersection of convex sets.

We also define

$$
\theta_{0}:=\sup \left\{\varangle\left(\gamma_{o p}^{\prime}(0), \gamma_{o q}^{\prime}(0)\right) ; p \in T_{0}, q \in S_{1}(p)\right\} .
$$

Therefore, $\varangle\left(v, \gamma_{o x}^{\prime}(0)\right) \leq \alpha+\theta_{0}$, for all $x \in D_{1}$. In fact, if $x \in D_{1}$, there exists $p \in T_{0}$ such that $x \in B\left(r_{1}\right) \backslash C_{1, p}$, which implies that $r_{0}+\varepsilon \phi\left(\rho_{p}(x)\right)<\rho(x)<r_{1}$, and then $x \in B_{1}(p)$, otherwise we would conclude that $r_{1}<r_{1}$. Hence

$$
\varangle\left(v, \gamma_{o x}^{\prime}(0)\right) \leq \varangle\left(v, \gamma_{o p}^{\prime}(0)\right)+\varangle\left(\gamma_{o p}^{\prime}(0), \gamma_{o x}^{\prime}(0)\right) \leq \alpha+\theta_{0} .
$$

We now proceed inductively in the following way: Assume that $C_{k}, r_{k}, D_{k}, T_{k}$ are defined for all $k \leq n-1, n \geq 1$, and that all $C_{k}$ 's are convex sets. By Lemma 3.6. there exists uniform $\varepsilon_{n}$ such that if $p \in S_{r_{n-1}}(o)$, the set $C_{n, p}:=\left\{\rho_{o}-\varepsilon_{n-1} f_{p} \leq\right.$ $\left.r_{n-1}\right\}$ is convex. Define

$$
\begin{aligned}
& \widetilde{C_{n}}:=\bigcap_{p \in T_{n-1}} C_{n, p}, \quad C_{n}:=\widetilde{C_{n} \backslash D_{n-1}, \quad r_{n}:=r_{n-1}+\varepsilon_{n-1},} \\
& D_{n}:=B_{r_{n}}(0) \backslash C_{n}, \quad T_{n}:=\overline{S_{r_{n}}(o) \backslash \partial C_{n}}, \\
& \theta_{n}:=\sup \left\{\varangle\left(\gamma_{o p}^{\prime}(0), \gamma_{o q}^{\prime}(0)\right) ; p \in T_{n}, q \in S_{1}(p)\right\} .
\end{aligned}
$$

Claim 1. $C_{n}$ is a convex set.

For, let $x, y \in C_{n}$ and assume, by contradiction, that $\gamma_{x y} \nsubseteq C_{n}$. Since $C_{n} \subset \widetilde{C_{n}}$, which is convex, it must occur that $\gamma_{x y} \cap D_{n-1} \neq \emptyset$. In particular, $\gamma_{x y}$ must intersect $\partial D_{n-1}$ at least twice. On the other hand, since $C_{n-1}$ is a convex set, $\gamma_{x y}$ cannot cross $\partial C_{n-1}$ twice, otherwise it would be contained in $C_{n-1} \subset C_{n}$. Hence $\gamma_{x y}$ must intersect $\partial D_{n-1} \backslash \partial C_{n-1}=\operatorname{int} T_{n-1}$. But in that case, $\gamma_{x y}$ would contain points of $B_{r_{n}}(o) \backslash \widetilde{C_{n}}$, which contradicts the convexity of $\widetilde{C_{n}}$. 
Claim 2. If $x \in D_{n}$, then $\varangle\left(v, \gamma_{o x}^{\prime}(0)\right) \leq \alpha+\sum_{i=0}^{n-1} \theta_{i}$.

In fact, the definition of $D_{n}$ implies that $D_{n}=D_{n-1} \cup\left(B_{r_{n}}(o) \backslash \widetilde{C_{n}}\right)$. If $x \in$ $D_{n-1}$, it is finished, since by induction it is possible to conclude that $\varangle\left(v, \gamma_{o y}^{\prime}(0)\right) \leq$ $\alpha+\sum_{i=0}^{n-2} \theta_{i}$ for all $y \in D_{n-1}$. Otherwise, there exists $p \in T_{n-1}$ such that $x \in$ $B_{r_{n}}(o) \backslash C_{n, p}$, which implies that $x \in B_{r_{n}}(o) \cap B_{1}(p)$ for some $p \in T_{n-1}$. Then it holds that

$$
\begin{aligned}
\varangle\left(v, \gamma_{o x}^{\prime}(0)\right) & \leq \varangle\left(v, \gamma_{o p}^{\prime}(0)\right)+\varangle\left(\gamma_{o p}^{\prime}(0), \gamma_{o x}^{\prime}(0)\right) \\
& \leq \varangle\left(v, \gamma_{o p}^{\prime}(0)\right)+\theta_{n-1} .
\end{aligned}
$$

On the other hand, it is easy to see that $T_{n-1} \subset \overline{D_{n-1}}$; hence

$$
\varangle\left(v, \gamma_{o p}^{\prime}(0)\right) \leq \alpha+\sum_{i=0}^{n-2} \theta_{i},
$$

which concludes the proof of Claim 2 .

Claim 3. Let

$$
C:=\bigcup_{n \geq 0} C_{n}, \quad D:=\bigcup_{n \geq 0} D_{n} .
$$

Then $C$ is a convex set and $M=C \cup D$.

Since by construction we have $C_{0} \subseteq C_{1} \subseteq C_{2} \subseteq \cdots$ and all $C_{n}$ 's are convex, it follows immediately that $C$ is convex. In order to prove that $M=C \cup D$, it is sufficient to show that $r_{n} \rightarrow+\infty$ as $n \rightarrow+\infty$ because $B_{r_{n}}(o)=C_{n} \cup D_{n}$. If we assume, by contradiction, that there exists $A>0$ such $r_{n} \leq A$ for all $n \geq 0$, we conclude that

$$
\varepsilon_{n}=\beta r_{n}^{1+\epsilon} e^{-k r_{n}} \geq \beta r_{0}^{1+\epsilon} e^{-k A}, \forall n \geq 0,
$$

and using that

$$
r_{n+1}=r_{n}+\varepsilon_{n}=r_{0}+\sum_{i=0}^{n} \varepsilon_{n},
$$

we conclude that $r_{n} \rightarrow+\infty$, which leads to an absurdity.

Because of Claim 3, to obtain (i) it suffices to prove that

$$
\partial_{\infty} D \subset\left\{\tilde{\gamma}(\infty) \mid \varangle_{o}(v, \tilde{\gamma}(\infty)) \leq 2 \alpha\right\} .
$$

For, notice that, at least formally, if $x \in D$,

$$
\varangle_{o}\left(v, \gamma_{o x}^{\prime}(0)\right) \leq \alpha+\sum_{n=0}^{+\infty} \theta_{n} .
$$

The only thing that remains to be proved is that the series converges to a constant $\leq \alpha$ if we choose $r_{0}$ correctly. The main idea is to estimate how many $r_{i}$ 's are on each interval in order to control, in some sense, the number of terms of the sum. For, let $t_{n}$ be the number of $r_{i}$ 's on the interval $I_{n}:=\left[r_{0}+n, r_{0}+n+1\right], n \geq 0$. Let $j_{n}$ be the index of the greatest $r_{i}$ on $I_{n}$. Since $\varepsilon_{i}$ is decreasing on $i$, we have

$$
\begin{aligned}
t_{n} \varepsilon_{j_{n}} & \leq \varepsilon_{j_{n}-1}+\varepsilon_{j_{n}-2}+\cdots+\varepsilon_{j_{n}-t_{n}} \\
& =\left(r_{j_{n}}-r_{j_{n}-1}\right)+\left(r_{j_{n}-1}-r_{j_{n}-2}\right)+\cdots+\left(r_{j_{n}+1-t_{n}}-r_{j_{n}-t_{n}}\right) \\
& =r_{j_{n}}-r_{j_{n}-t_{n}} \leq\left(r_{0}+n+1\right)-\left(r_{0}+n\right)=1 .
\end{aligned}
$$


It implies that

$$
t_{n} \leq \varepsilon_{j_{n}}^{-1} \leq \frac{e^{k\left(r_{0}+n+1\right)}}{\beta\left(r_{0}+n\right)^{1+\epsilon}},
$$

where the last inequality is true since $r_{0}+n \leq r_{j_{n}} \leq r_{0}+n+1$.

Combining the above estimative with Lemma 3.8 (see Section 3.1), we obtain that

$$
\sum_{i=0}^{\infty} \theta_{i}=\sum_{n=0}^{\infty} \sum_{r_{i} \in I_{n}} \theta_{i} \leq \sum_{n=0}^{\infty} t_{n} C e^{-k\left(r_{0}+n\right)} \leq \frac{C e}{\beta} \sum_{n=0}^{\infty} \frac{1}{\left(r_{0}+n\right)^{1+\epsilon}} .
$$

It is then clear that with a correct choice of $r_{0}$ it is possible to obtain $\theta<2 \alpha$.

To prove (ii), let $\tilde{\gamma}$ be a geodesic ray from $o$ satisfying $\varangle_{o}\left(\tilde{\gamma}^{\prime}(0), v\right)<\alpha$. Then $\tilde{\gamma}$ must intersect $T_{0}$ at distance $r_{0}$, going out of $C$. Since by construction it holds that $\partial C \supseteq T_{0}$ and $C$ is convex, $\tilde{\gamma}$ cannot intersect $C$ after a distance $r_{0}$.

\subsection{Appendix.}

Lemma 3.7. Let $M$ be a rotationally symmetric manifold, with metric given by

$$
d r^{2}+f(r)^{2}\left(d \theta^{2}+\sin ^{2} \theta d \varphi^{2}\right)
$$

where $f:[0,+\infty) \rightarrow \mathbb{R}$ is a smooth function that satisfies $f(0)=0, f^{\prime}(0)=1$ and $r$ is the distance function to the center o of $M$. Suppose that $K_{M} \leq-k^{2}<0$. Then for all $r \geq 0$,

$$
\frac{f^{\prime}(r)}{f(r)} \geq k \operatorname{coth}(k r), \quad f(r) \geq \frac{\sinh (k r)}{k} \text { and } f^{\prime}(r) \geq \cosh (k r) .
$$

Proof. Let $p \in M$ such that $r(p)=r$. Then it is well known that the sectional curvature of a 2-plane generated by $\gamma_{o p}^{\prime}(r)$ and any other vector is given by $-f^{\prime \prime}(r) / f(r)$. Let $g(r):=(1 / k) \sinh (k r)$. Since $K_{M} \leq-k^{2}$, it occurs that

$$
\begin{array}{r}
\frac{f^{\prime \prime}(r)}{f(r)} \geq k^{2}=\frac{g^{\prime \prime}(r)}{g(r)} \Rightarrow f^{\prime \prime}(r) g(r)-g^{\prime \prime}(r) f(r) \geq 0 \\
\Rightarrow\left(f^{\prime}(r) g(r)-g^{\prime}(r) f(r)\right)^{\prime} \geq 0 \Rightarrow f^{\prime}(r) g(r)-g^{\prime}(r) f(r) \geq 0 ;
\end{array}
$$

therefore

$$
\frac{f^{\prime}(r)}{f(r)} \geq \frac{g^{\prime}(r)}{g(r)}=k \operatorname{coth} k r
$$

and also

The last inequality implies that

$$
\left(\frac{f(r)}{g(r)}\right)^{\prime} \geq 0
$$

$$
\frac{f(r)}{g(r)} \geq \lim _{r \rightarrow 0^{+}} \frac{f(r)}{g(r)}=1 \Rightarrow f(r) \geq g(r)=\frac{\sinh k r}{k} .
$$

Using this fact on (31), it is possible to conclude that $f^{\prime}(r) \geq g^{\prime}(r)=\cosh k r$, which concludes the proof.

Lemma 3.8. Let $M$ be a Hadamard manifold with $K_{M} \leq-k^{2}$, o $\in M$ and $p \in M$ such that $\rho_{o}(p)=R$, with $R \geq \tilde{r}$, where $\tilde{r}$ is a universal constant. Then

$$
\theta_{R}:=\max \left\{\varangle\left(\gamma_{o p}^{\prime}(0), \gamma_{o q}^{\prime}(0)\right) ; q \in S_{1}(p)\right\} \leq 2 \frac{\sinh k}{\sinh k R} .
$$

In particular, there exists a universal constant $C>0$ such that $\theta_{R} \leq C e^{-k R}$. 
Proof. By Toponogov's Theorem, it suffices to prove that the estimative for $\theta_{R}$ holds in the particular case that $M=\mathbb{H}^{2}\left(-k^{2}\right)$, the hyperbolic plane of sectional curvature $-k^{2}$. Let $\mathbb{D}^{2}$ be the unitary disc $\left\{(x, y) \in \mathbb{R}^{2} ; x^{2}+y^{2}<1\right\}$ endowed with the conformal metric

$$
\langle u, v\rangle_{(x, y)}=\frac{4\langle u, v\rangle_{e}}{k^{2}\left(1-x^{2}-y^{2}\right)^{2}}
$$

where $\langle\cdot, \cdot\rangle_{e}$ denotes the Euclidean metric.

Since the hyperbolic space is homogeneous, we may assume without loss of generality that $p$ has Euclidean coordinates $(a, 0), a>0$. An immediate consequence of the expression of the metric is that $a=\tanh (k R / 2)$. We observe that the hyperbolic and Euclidean angles at $o=(0,0)$ coincide, because the hyperbolic metric is conformal to the Euclidean one. Therefore, to compute $\theta_{R}$ it suffices to compute the Euclidean maximal angle of the circle $\mathbb{B}$ of hyperbolic center $(a, 0)$ and hyperbolic radius 1 . It is easy to see that $\theta_{R}$ is then given by

$$
\sin \theta_{R}=\frac{\text { Euclidean radius of } \mathbb{B}}{\text { Euclidean norm of the Euclidean center of } \mathbb{B}} .
$$

It then remains to compute the Euclidean radius and center of $\mathbb{B}$. For, notice that if we write $z=x+i y$, the Möebius transformation

$$
g(z):=\frac{z+a}{a z+1}
$$

is an isometry of $\mathbb{D}^{2}$ that maps the horizontal axis $\{y=0\}$ onto itself and maps the hyperbolic circle $\widetilde{\mathbb{B}}$ centered at $o$ of radius 1 (which is the Euclidean circle centered at $(0,0)$ of radius $b:=\tanh (k / 2)$ on the hyperbolic circle centered at $(a, 0)$ with hyperbolic radius 1). Observe that the Euclidean and hyperbolic radius and center do not coincide. However, by the expression of $g$ it is easy to see that $g(-b)$ and $g(b)$ form a diameter of $\mathbb{B}$, from which we obtain after straightforward computations that

$$
\begin{aligned}
\sin \theta_{R} & =\frac{g(b)-g(-b)}{g(b)+g(b)}=\frac{b\left(1-a^{2}\right)}{a\left(1-b^{2}\right)} \\
& =\frac{\tanh (k / 2)\left(1-\tanh ^{2}(k R / 2)\right)}{\tanh (k R / 2)\left(1-\tanh ^{2}(k / 2)\right)}=\frac{\tanh (k / 2) \cosh ^{2}(k / 2)}{\tanh (k R / 2) \cosh ^{2}(k R / 2)} \\
& =\frac{\sinh (k / 2) \cosh (k / 2)}{\sinh (k R / 2) \cosh (k R / 2)}=\frac{\sinh k}{\sinh k R}
\end{aligned}
$$

and then

$$
\theta_{R}=\arcsin \left(\frac{\sinh k}{\sinh k R}\right)
$$

and if $\sinh ^{2} k / \sinh ^{2} k R \leq 3 / 4$, we have $\sqrt{1-\sinh ^{2} k / \sinh ^{2} k R} \geq 1 / 2$, which implies that

$$
\arcsin \frac{\sinh k}{\sinh k R}=\int_{0}^{\frac{\sinh k}{\sinh k R}} \frac{d t}{\sqrt{1-t^{2}}} \leq \int_{0}^{\frac{\sinh k}{\sinh k R}} 2 d t
$$

which gives the result.

Furthermore, if $R \geq(\ln 2) / 2 k$, we obtain that $\sinh k R \geq e^{k R} / 4$, and hence if we write $C:=8 \sinh (k)$, we obtain the desired estimative, with $\tilde{r}$ satisfying both conditions $\sinh ^{2} k \tilde{r} \geq 4 \sinh ^{2}(k) / 3$ and $\tilde{r} \geq(\ln 2) / 2 k$. 


\section{REFERENCES}

[Anc] Alano Ancona, Convexity at infinity and Brownian motion on manifolds with unbounded negative curvature, Rev. Mat. Iberoamericana 10 (1994), no. 1, 189-220, DOI 10.4171/RMI/150. MR 1271762 (95a:58132)

[And] Michael T. Anderson, The Dirichlet problem at infinity for manifolds of negative curvature, J. Differential Geom. 18 (1983), no. 4, 701-721 (1984). MR.730923 (85m:58178)

[B1] Albert Borbély, A note on the Dirichlet problem at infinity for manifolds of negative curvature, Proc. Amer. Math. Soc. 114 (1992), no. 3, 865-872, DOI 10.2307/2159417. MR:1069289 (92f:58180)

[B2] Albert Borbély, The nonsolvability of the Dirichlet problem on negatively curved manifolds, Differential Geom. Appl. 8 (1998), no. 3, 217-237, DOI 10.1016/S0926-2245(98)00008-4. MR.1629348 (99j:53043)

[C] Hyeong In Choi, Asymptotic Dirichlet problems for harmonic functions on Riemannian manifolds, Trans. Amer. Math. Soc. 281 (1984), no. 2, 691-716, DOI 10.2307/2000080. MR.722769 (85b:53040)

[DHL] Marcos Dajczer, Pedro A. Hinojosa, and Jorge Herbert de Lira, Killing graphs with prescribed mean curvature, Calc. Var. Partial Differential Equations 33 (2008), no. 2, 231-248, DOI 10.1007/s00526-008-0163-8. MR2413108 (2009m:53154)

[DLR] M. Dajczer, J. H. de Lira, and J. Ripoll, An interior gradient estimate for the mean curvature equation of Killing graphs, preprint.

[EFR] Nedir do Espírito-Santo, Susana Fornari, and Jaime B. Ripoll, The Dirichlet problem for the minimal hypersurface equation in $M \times \mathbb{R}$ with prescribed asymptotic boundary (English, with English and French summaries), J. Math. Pures Appl. (9) 93 (2010), no. 2, 204-221, DOI 10.1016/j.matpur.2009.08.002. MR2584742 (2011e:35138)

[EO] P. Eberlein and B. O'Neill, Visibility manifolds, Pacific J. Math. 46 (1973), 45-109. MR0336648 (49 \#1421)

[GR] José A. Gálvez and Harold Rosenberg, Minimal surfaces and harmonic diffeomorphisms from the complex plane onto certain Hadamard surfaces, Amer. J. Math. 132 (2010), no. 5, 1249-1273, DOI 10.1353/ajm.2010.0001. MR2732346 (2011j:53012)

[GT] D. Gilbarg and N. Trudinger, Elliptic Partial Differential Equations of Second Order, Springer, Berlin, 1998.

[Ho] I. Holopainen, Nonsolvabily of the asymptotic Dirichlet problem for the p-Laplacian on Cartan-Hadamard manifolds, to appear in Advances in Calculus of Variations.

[HoR] I. Holopainen and J. Ripoll, Nonsolvability of the asymptotic Dirichlet problems for some quasilinear elliptic PDE on Hadamard manifolds, to appear in Revista Matematica Iberoamericana.

[HoV] Ilkka Holopainen and Aleksi Vähäkangas, Asymptotic Dirichlet problem on negatively curved spaces, J. Anal. 15 (2007), 63-110. MR2554093 (2010j:58056)

[Hs] Elton P. Hsu, Brownian motion and Dirichlet problems at infinity, Ann. Probab. 31 (2003), no. 3, 1305-1319, DOI 10.1214/aop/1055425781. MR1988474(2004e:58063)

[KN] Brett Kotschwar and Lei Ni, Local gradient estimates of p-harmonic functions, 1/H-flow, and an entropy formula (English, with English and French summaries), Ann. Sci. Éc. Norm. Supér. (4) 42 (2009), no. 1, 1-36. MR2518892 (2010g:53121)

[Ne] R. W. Neel, Brownian motion and the Dirichlet problem at infinity on two-dimensional Cartan-Hadamard manifolds, Potential Anal. 41 (2014), no. 2, 443-462. MR3232032

[RS] Marco Rigoli and Alberto G. Setti, Liouville type theorems for $\phi$-subharmonic functions, Rev. Mat. Iberoamericana 17 (2001), no. 3, 471-520, DOI 10.4171/RMI/302. MR.1900893 (2003b:58049)

[RT] Jaime Ripoll and Miriam Telichevesky, Complete minimal graphs with prescribed asymptotic boundary on rotationally symmetric Hadamard surfaces, Geom. Dedicata 161 (2012), 277-283, DOI 10.1007/s10711-012-9706-4. MR2994043

[SY] R. Schoen and S.-T. Yau, Lectures on differential geometry, Conference Proceedings and Lecture Notes in Geometry and Topology, I, International Press, Cambridge, MA, 1994. Lecture notes prepared by Wei Yue Ding, Kung Ching Chang [Gong Qing Zhang], Jia Qing Zhong and Yi Chao Xu; translated from the Chinese by Ding and S. Y. Cheng; preface translated from the Chinese by Kaising Tso. MR.1333601 (97d:53001) 
[S] Joel Spruck, Interior gradient estimates and existence theorems for constant mean curvature graphs in $M^{n} \times \mathbf{R}$, Pure Appl. Math. Q. 3 (2007), no. 3, Special Issue: In honor of Leon Simon, 785-800. MR2351645 (2009b:58025)

[V] A. Vähäkangas, Dirichlet problem at infinity for p-harmonic functions on negatively curved spaces, Academic Dissertation, Department of Mathematics and Statistics, Faculty of Science, University of Helsinki, 2008.

Instituto de Matemática, Universidade Federal do Rio Grande do Sul, Av. Bento Gonçalves, 9500, CeP 91540-000, Porto Alegre, Rio Grande do Sul, Brasil

E-mail address: jaime.ripoll@ufrgs.br

Instituto de Matemática, Universidade Federal do Rio Grande do Sul, Av. Bento Gonçalves, 9500, CEP 91540-000, Porto Alegre, Rio Grande do Sul, Brasil

E-mail address: miriam.telichevesky@ufrgs.br 